University of Nebraska - Lincoln

DigitalCommons@University of Nebraska - Lincoln

June 2005

\title{
Nanoscale polarization relaxation in a polycrystalline ferroelectric thin film: Role of local environments
}

V. Nagarajan

University of New South Wales, Sydney

S. Aggarwal

Texas Instruments Incorporated, 13560 North Central Expressway, MS 3736 Dallas, Texas

Alexei Gruverman

University of Nebraska-Lincoln, agruverman2@unl.edu

R. Ramesh

University of California, at Berkeley

R. Waser

Institute for Solid State Physics and CNI, Forschungszentrum Juelich, D 52425 Germany

Follow this and additional works at: https://digitalcommons.unl.edu/physicsgruverman

Part of the Physics Commons

Nagarajan, V.; Aggarwal, S.; Gruverman, Alexei; Ramesh, R.; and Waser, R., "Nanoscale polarization relaxation in a polycrystalline ferroelectric thin film: Role of local environments" (2005). Alexei Gruverman Publications. 19.

https://digitalcommons.unl.edu/physicsgruverman/19

This Article is brought to you for free and open access by the Research Papers in Physics and Astronomy at DigitalCommons@University of Nebraska - Lincoln. It has been accepted for inclusion in Alexei Gruverman Publications by an authorized administrator of DigitalCommons@University of Nebraska - Lincoln. 


\title{
Nanoscale polarization relaxation in a polycrystalline ferroelectric thin film: Role of local environments
}

\author{
V. Nagarajan ${ }^{\mathrm{a})}$ \\ School of Materials Science and Engineering, University of New South Wales, Sydney NSW 2052 \\ S. Aggarwal \\ Texas Instruments Incorporated, 13560 North Central Expressway, MS 3736 Dallas, Texas 75243
}

A. Gruverman

Department of Materials Science and Engineering, North Carolina State University, Raleigh, North Carolina 27695-7920

\author{
R. Ramesh \\ Department of Materials Science, University of California, at Berkeley, Berkeley, California 94720 \\ R. Waser \\ Institute for Solid State Physics and CNI, Forschungszentrum Juelich, D 52425 Germany
}

(Received 8 February 2005; accepted 19 May 2005; published online 24 June 2005)

\begin{abstract}
In this letter, we report on the study of nanoscale polarization relaxation phenomena in polycrystalline $\mathrm{PbZr}_{0.4} \mathrm{Ti}_{0.6} \mathrm{O}_{3}$ films. Piezoresponse force microscopy (PFM) images of the as-grown sample reveal grains with a range of contrast, from fully white to gray to fully black. It is shown that this local change in the contrast (magnitude) of the piezoresponse from grain to grain can be attributed to the crystallographic orientation within each grain. PFM-based relaxation experiments show that the rate of relaxation is different for each grain, furthermore it is strongly dependent on the tilt of individual crystallographic orientation with respect to the polar axis. Strongly tilted away nonpolar axis grains show a much stronger decay of the polarization compared to polar axis-oriented grains. Therefore, for an ensemble of grains under a common top electrode, the relaxation events would first take place in grains, which are nonpolar axis oriented. (C) 2005 American Institute of Physics. [DOI: 10.1063/1.1977183]
\end{abstract}

The properties of ferroelectric materials at the nanoscale have attracted considerable attention over the past few years. ${ }^{1,2}$ Of equal importance is the ability to measure and characterize ferroelectric devices at the nanoscale and hence electrical characterization with scanned probe microscopy, especially piezoresponse force microscopy (PFM), has tremendously progressed. This technique has been used in an exhaustive manner to understand nanoscale ferroelectric domain structure, ${ }^{3-9}$ polarization relaxation, ${ }^{10-14}$ domain-wall creep, ${ }^{15}$ piezoelectric properties, ${ }^{16-20}$ scaling effects, ${ }^{17,21,22}$ reliability issues, ${ }^{23,24}$ and properties of individual grains. ${ }^{25}$ Although there are reports that correlate piezoresponse of the ferroelectric thin film to the possible crystallographic orientations, ${ }^{7,26,27}$ there is scarce information on how the crystallography of each individual grain affects the polarization relaxation. In this letter, we use the technique of PFM, first to identify polar axis- and nonpolar axis-oriented grains, and then further study the polarization relaxation of such individual grains. We show that the larger the deviation from the polar axis within a given grain from the film surface normal, the faster the rate of the relaxation. Therefore, for an ensemble of grains under a common top electrode [typically the scenario in a commercial ferroelectric random access memory (FRAM) capacitor], our results suggest that the relaxation events would first take place in grains whose polar axis is away from the film normal.

\footnotetext{
${ }^{a)}$ Electronic mail: nagarajan@unsw.edu.au
}

$\mathrm{PbZr}_{0.4} \mathrm{Ti}_{0.6} \mathrm{O}_{3}$ (PZT) films $70 \mathrm{~nm}$ thick grown on $\mathrm{IrO}_{2} / \mathrm{Ir} / \mathrm{TiAlN}$ buffered Si by metalorganic chemical vapor deposition process were used for this study. X-ray diffraction of the film identified the structure of the PZT layer to be tetragonal and with a mixture of (100), (110), and (111) orientations. Further details on the film processing and typical macroscopic ferroelectric properties can be found elsewhere. ${ }^{28}$ PFM experiments were performed on a Veeco Nanoscope IV system with standard Pt-Ir-coated tips.

Figure 1(a) is a plot of the effective out-of-plane piezoelectric coefficient $\left(d_{z z}\right)$ as a function of the angle of deviation between the polar axis and the film surface normal. The exact correlation for a tetragonal system between the $d_{z z}$ and the $d_{33}$ is given as: ${ }^{26,27}$

$$
d_{z z}(\theta)=\left(d_{31}+d_{15}\right) \sin ^{2} \theta \cos \theta+d_{33} \cos ^{3} \theta .
$$

Equation (1) shows that $d_{z z}$ would be maximum for those grains oriented along the (001) orientation (i.e., with an angle $\theta=0^{\circ}$ or $180^{\circ}$ ), equal to the $d_{33}$, and for all other orientations, the magnitude of $d_{z z}$ (and, hence, the piezoresponse contrast) would be dependent on $\theta$. Figure 1(b) is a typical vertical PFM (VPFM) image of the ferroelectric film. It shows a number of grains, each about $50-100 \mathrm{~nm}$ in size. We immediately observe a wide range of contrast in the grain; from fully white (Grain A) to gray (Grain B) to fully black (Grain C). We did not observe any $90^{\circ}$ domain twins within each grain, unlike the scenario in $\mathrm{PbTiO}_{3}$ films. ${ }^{6}$ From the phase image acquired concurrent with the VPFM, we do not observe any $180^{\circ}$-type phase boundaries within regions of the same contrast, unlike those reported by Stolichnov ${ }^{23}$ 

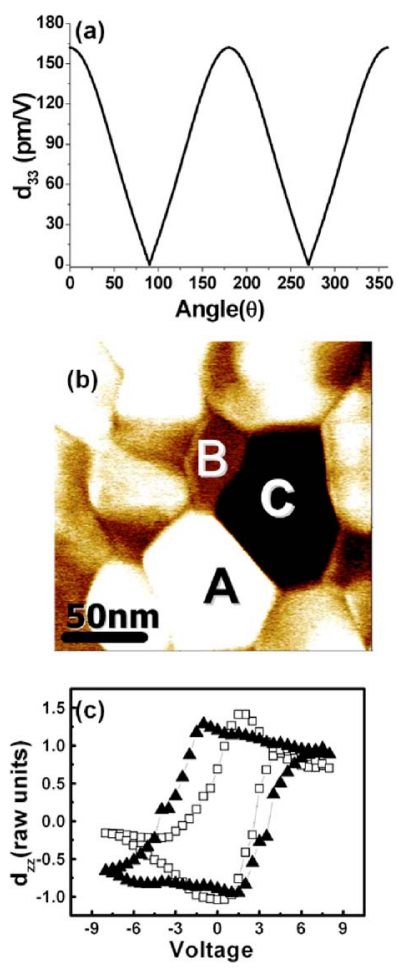

FIG. 1. (Color online) (a) Effective out-of-plane piezoresponse, $d_{z z}$ as a function of the angle made by the crystallographic orientation with the polarization vector. For a full 3D plot refer to Uchino et al. (Ref. 26) and Harnagea et al. (Ref. 27). (b) Typical VPFM image of the PZT film used in this study. Three grains are marked, fully white (A), gray (B) and fully black (C). (c) Piezoloops for Grains A (open squares) and B (solid triangles).

and Gruverman et $a l .{ }^{7}$ We thus conclude that the difference in the magnitude (and hence contrast) in the piezoresponse between the individual grains is due to different crystallographic orientations. The piezoresponse of Grains $\mathrm{A}$ and $\mathrm{C}$ in the VPFM image was maximum, and zero in the lateral PFM (LPFM) image; these grains are inferred to be (001) oriented. Conversely, there are some grains, which show zero response in the VPFM but show maximum response in the LPFM. In such grains, the polarization is oriented fully in plane, i.e., along (010) or (100) orientation. On the other hand, majority of the grains show gray contrast (hereafter referred to as gray grains) in both, the VPFM and LPFM response, with the intensity ranging from 50 to $65 \%$ of the maximum (for e.g., Grain B). These grains have their polarization vector at an angle with the film normal. Figure 1(c) shows a piezoresponse loop measured for a fully white grain and a typical gray grain. We see that the maximum response for the white grain (open symbols) is larger than the gray grain (closed symbols) at remanence (zero fields). This agrees very well with the imaging data where gray grains (nonpolar axis oriented) show a lower piezoresponse than the white grains. In addition, the coercive voltage for the gray grain is significantly larger than that of the white grain similar to studies on SBT films by Kalinin et al. ${ }^{25}$

To understand the impact of such various grain orientations on the polarization relaxation, we performed PFMbased relaxation experiments. Figure 2 is a series of VPFM images of a region after writing a submicron bit with an applied voltage of $-4 \mathrm{~V}$. Figure $2(\mathrm{a})$ is an image of the virgin surface; Fig. 2(b) is a scan of the central region with the bit in the "as-written" state. After an application of dc bias, the
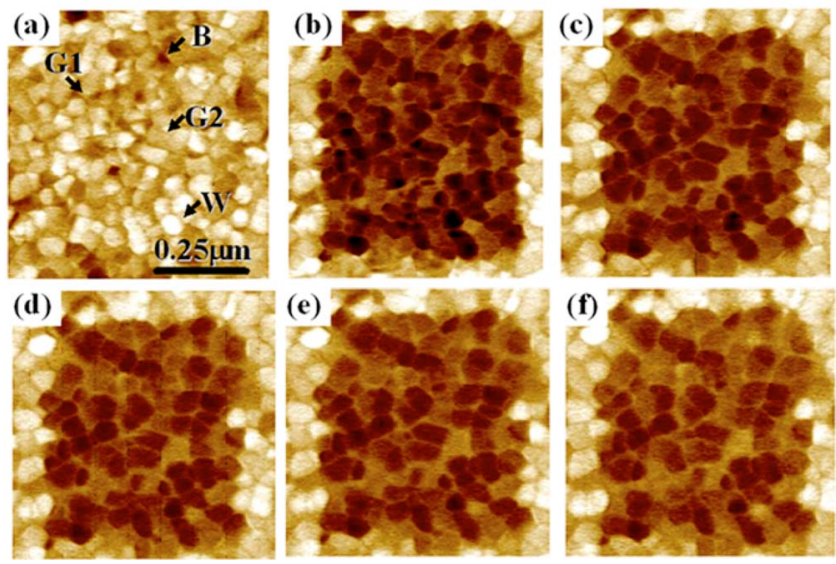

FIG. 2. (Color online) (a)-(f) PFM images as a function of time for a $-4 \mathrm{~V}$ write. (a) An image of the sample as grown. (b) PFM image of the central region in (a) after writing it with $-4 \mathrm{~V}$ dc bias. (c)-(f) PFM images after $512,8192,32000$, and $97000 \mathrm{~s}$, respectively. The arrows in the figure show four grains (W-White, B-Black, G1-Gray 1, G2-Gray 2) for which time dependent piezoresponse is plotted in Fig. 3.

contrast is changed to black, in comparison to a lighter unaffected background. Figures 2(c)-2(f) are images taken after several time intervals, from $512 \mathrm{~s}$ to $97000 \mathrm{~s}$. In order to eliminate tip wear-induced effects and ensure a rigorous calibration, a marker grain was chosen which was significantly away from the poled region, and thus not affected by the applied voltage. For each image, the piezoresponse was calibrated with respect to this marker grain. A primary observation is that, unlike our previous report on epitaxial PZT films ${ }^{11}$ no significant lateral shrinkage of the written bit is observed.

In contrast, for grains within the written bit, a monotonic decrease in the reduction of the vertical piezoresponse signal was observed as a function of time, indicating vertical back switching similar to previously published reports by other groups. ${ }^{14,29}$ Figure 3 (a) shows the piezoresponse measured as a function of time for four grains-Black (B), White (W), Gray-1 (G1), and Gray-2 (G2), from Fig. 2. Each of the grains were approximately $50-100 \mathrm{~nm}$ in size. In order to plot them on the same scale, the piezoresponse was normalized with respect to the original piezoresponse for each individual grain. The solid lines in each plot are fits to a stretch exponential of the form:

$$
P=P_{o} \exp \left(-\left(\frac{t}{\tau}\right)\right)^{n}
$$

where $n$ is the dimensional constant and $\tau$ is the characteristic time. The grain which was fully black or fully white in
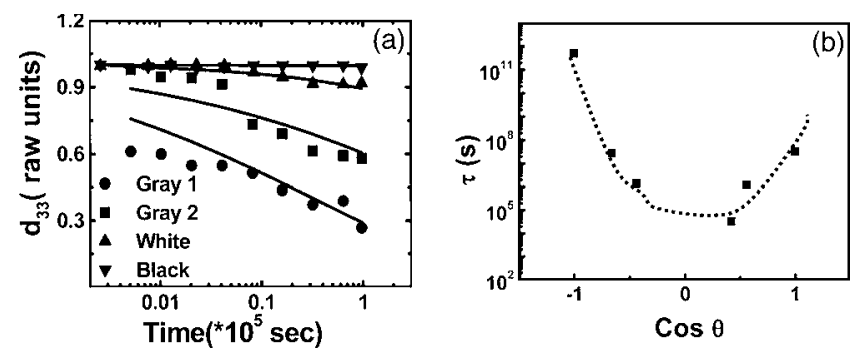

FIG. 3. (a) Piezoresponse as a function of time for B, W, G1 and G2 grains. The solid lines in the figure are fits to stretch exponential in Eq. (2). (b) Relaxation time $\tau$ as a function of the angle of deviation. It shows that as one deviates from the polar axis, the relaxation proceeds faster. The dotted line is a guide for the eyes. 

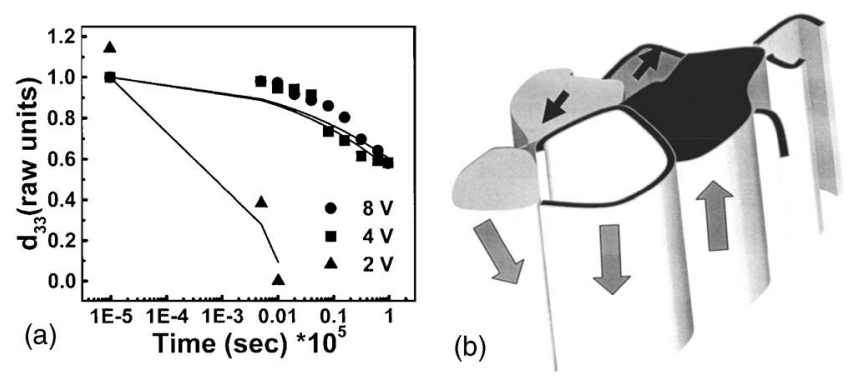

FIG. 4. (a) The polarization relaxation dependence for typical gray grains as a function of three write voltages: $-2 \mathrm{~V},-4 \mathrm{~V}$, and $8 \mathrm{~V}$. (b) Sketch of the PFM response of the grains in the film.

the original state, shows only marginal relaxation with the signal dropping by less than $5 \%$. We note that for both grains the exponent is constant, $n$ to be $\sim 0.25$ although $\tau$ for the black grain $\left(\approx 5 \times 10^{11} \mathrm{~s}\right)$ was significantly larger compared to the white grain $\left(\approx 3.25 \times 10^{7} \mathrm{~s}\right)$. On the other hand, the relaxation behaviors of grains that were originally gray in contrast are markedly different. These grains in general relax much quicker than the fully black or fully white grains. Although the value of $n(\approx 0.25)$ was not different from the $(001)$ grains, the time constants were markedly worse, with $\tau$ dropping to only $10^{3} \mathrm{~s}$. Since these grains form a majority of the film, it is hence not surprising that they would dominate the relaxation process for an ensemble of grains. In Fig. 3(b), we plot the characteristic time, $\tau$, measured for the $-4 \mathrm{~V}$ write experiment plotted as a function of the angle of deviation $[\cos (\theta)]$ for many grains. The line is a guide for the eyes. For grains whose polar axis are $(001)$ or $(00 \overline{1})$ oriented (at the opposite ends of the $x$ axis), $\tau$ is very high. As one deviates further away from the polar axis, i.e., moves toward lower $\cos (\theta), \tau$ changes from $10^{11} \mathrm{~s}$ to only $10^{3} \mathrm{~s}$. Thus, a systematic drop in $\tau$ is observed, commensurate with the angle of deviation $\theta$.

An explanation of the observed accelerated polarization relaxation in the nonpolar grains could be that the effective switching field is lower than that compared for polar axis grains. This can be deduced form the $d_{33}$ loops in Fig. 1(a), where in the polar axis Grain A has a much lower coercive voltage compared to the nonpolar axis Grain B. Figure 4(a) plots the polarization relaxation dependence for typical gray grains as a function of three write voltages: $-2 \mathrm{~V},-4 \mathrm{~V}$, and $8 \mathrm{~V}$. As $-2 \mathrm{~V}$ is below the coercive voltage the grain relaxes much faster. However, the time dependent behavior for the -4 and $-8 \mathrm{~V}$ are very similar. It can be concluded that the observed phenomena cannot be explained because of a lower switching field-although this may play a role in the complete picture.

In order to understand this preferential backswitching shown by the nonpolar grains, we must look at the electrostatics of the domain reversal process. Figure 4 is a threedimensional (3D) sketch of the polarization derived from a typical PFM image. It shows that for fully black or white grains, the there is no polarization charge across the grain boundary and hence the $\operatorname{div} P=0$. However, for nonpolar axis-oriented grains, the polarization makes finite angles with the neighboring grains creating a nonzero charge at the grain boundary. This would create a strong depoling field at these interfaces, which has its source at the divergence of the polarization across the grain boundary. It is obvious that the stronger the deviation of the polarization across the grain boundary, the stronger the depolarization field. Thus, larger values of $\theta$, would lead to larger divergence at this interface and, consequently, a larger depolarizing field. This depolarizing field would favor the immediate nucleation of reverse domains. $^{13,30}$

This work was supported by NSF MRSEC DMR 008008. One of the authors (V.N.) acknowledges the support of the Alexander von Humboldt Foundation for his stay at FZJuelich.

${ }^{1}$ M. Alexe and A. Gruverman, Nanoscale Characterisation of Ferroelectric Materials (Springer, Berlin, 2004).

${ }^{2}$ H. Ishiwara, M. Okuyama, and Y. Arimoto, Ferroelectric Random Access Memories: Fundamentals and Applications, (Springer, Berlin, 2004), Vol. 93.

${ }^{3}$ A. Gruverman, O. Auciello, and H. Tokumoto, Annu. Rev. Mater. Sci. 28, 101 (1998).

${ }^{4}$ L. M. Eng, H.-J. Guentherodt, G. Rosenman, A. Skliar, M. Oron, M. Katz, and D. Eger, J. Appl. Phys. 83, 5973 (1998).

${ }^{5}$ S. Hong, J. Woo, H. Shin, J. U. Jeon, Y. E. Pak, E. L. Colla, N. Setter, E. Kim, and K. No, J. Appl. Phys. 89, 1377 (2001).

${ }^{6}$ A. Roelofs, U. Boettger, R. Waser, F. Schlaphof, S. Trogisch, and L. M. Eng, Appl. Phys. Lett. 77, 3444 (2000).

${ }^{7}$ B. J. Rodriguez, A. Gruverman, A. I. Kingon, and R. J. Nemanich, J. Appl. Phys. 95, 1958 (2004).

${ }^{8}$ S. Hong, B. Escabart, E. L. Colla, and N. Setter, Appl. Phys. Lett. 84, $2382(2004)$.

${ }^{9}$ S. V. Kalinin and D. A. Bonnell, Phys. Rev. B 65, 125408 (2002).

${ }^{10}$ C. S. Ganpule, V. Nagarajan, H. Li, A. S. Ogale, D. E. Steinhaueur, S. A. Aggarwal, E. Williams, and R. Ramesh, Appl. Phys. Lett. 77, 292 (2000).

${ }^{11}$ C. S. Ganpule, A. L. Roytburd, V. Nagarajan, B. K. Hill, S. B. Ogale, E. D. Williams, R. Ramesh, and J. F. Scott, Phys. Rev. B 65, 014101-7 (2001).

${ }^{12}$ C. S. Ganpule, V. Nagarajan, S. P. Alpay, A. Royturd, and R. Ramesh, J. Appl. Phys. 91, 1477 (2002).

${ }^{13}$ A. Roelofs, N. A. Pertsev, R. Waser, F. Schlaphof, L. M. Eng, C. Ganpule, V. Nagarajan, and R. Ramesh, Appl. Phys. Lett. 80, 1424 (2002).

${ }^{14}$ D. S. Fu, K. Suzuki, K. Kato, and H. Kuzuki, Appl. Phys. Lett. 82, 2130 (2003).

${ }^{15}$ T. Tybell, P. Paruch, T. Giamarchi, and J.-M. Triscone, Phys. Rev. Lett. 89, 97601-1 (2002).

${ }^{16}$ J. A. Christman, J. R. R. Woolcott, A. I. Kingon, and R. J. Nemanich, Appl. Phys. Lett. 73, 3851-53 (1998).

${ }^{17}$ C. S. Ganpule, A. Stanishevsky, Q. Su, S. Aggarwal, J. Melngailis, E. Williams, and R. Ramesh, Appl. Phys. Lett. 75, 409 (1999).

${ }^{18}$ V. Nagarajan, A. Roytburd, A. Stanishevsky, S. Prasertchoung, T. Zhao, L. Chen, J. Melngailis, O. Auciello, and R. Ramesh, Nat. Mater. 2, 43 (2003).

${ }^{19}$ V. Nagarajan, A. Stanishevsky, T. Zhao, L. Chen, J. Melngailis, A. R. Royturd, and R. Ramesh, Appl. Phys. Lett. 81, 4215 (2002).

${ }^{20}$ C. Harnagea, M. Alexe, D. Hesse, and A. Pignolet, Appl. Phys. Lett. 83, 338 (2003)

${ }^{21}$ T. Tybell, C. H. Ahn, and J.-M. Triscone, Appl. Phys. Lett. 75, 856 (1999).

${ }^{22}$ T. Tybell, C. H. Ahn, and J.-M. Triscone, Appl. Phys. Lett. 72, 1454 (1998).

${ }^{23}$ E. L. Colla, I. Stolichnov, P. E. Bradley, and N. Setter, Appl. Phys. Lett. 82, 1604 (2003).

${ }^{24}$ I. Stolichnov, E. Colla, A. Tagantsev, S. S. N. Bharadwaja, S. Hong, N. Setter, J. S. Cross, and M. Tsukada, Appl. Phys. Lett. 80, 4804 (2002).

${ }^{25}$ S. V. Kalinin, A. Gruverman, and D. Bonnell, Appl. Phys. Lett. 85, 795 (2004).

${ }^{26}$ X. H. Du, U. Belegundu, and K. Uchino, Jpn. J. Appl. Phys., Part 1 36, 5580 (1997).

${ }^{27}$ C. Harnagea, A. Pignolet, M. Alexe, and D. Hesse, Integr. Ferroelectr. 38, 23 (2001).

${ }^{28}$ S. R. Gilbert, S. Hunter, D. Ritchey, C. Chi, D. V. Taylor, J. Amano, S. Aggarwal, T. S. Moise, T. Sakoda, S. R. Summerfelt, K. K. Singh, C. Kazemi, D. Carl, and B. Bierman, J. Appl. Phys. 93, 1713 (2003).

${ }^{29}$ S. Hong, E. L. Colla, E. Kim, D. V. Taylor, A. K. Tagantsev, P. Muralt, K. No, and N. Setter, J. Appl. Phys. 86, 607 (1999).

${ }^{30}$ R. Landauer, J. Appl. Phys. 28, 227 (1957). 